\title{
ASPECTOS ECOFISIOLÓGICOS DE LA FEIJOA (Acca sellowiana Berg) BAJO CONDICIONES DE RIEGO Y DÉFICIT HÍDRICO
}

\section{ECOPHYSIOLOGICAL ASPECTS OF FEIJOA (Acca sellowiana Berg) UNDER IRRIGATION AND HYDRIC DEFFICIENCY}

\author{
Fernando Javier Peña Baracaldo ${ }^{1}$, Marco Cabezas Gutiérrez ${ }^{2}$
}

\begin{abstract}
${ }^{1}$ Ing. Agrónomo, M.Sc. Docente-Investigador, Programa de Ingeniería Agronómica, Universidad de Ciencias Aplicadas y Ambientales U.D.C.A, calle 222 No. 55-37, Bogotá, D.C.-Colombia, e-mail: fjpb74@ hotmail.com; ${ }^{2}$ Ingeniero Agrónomo, M.Sc., cPh.D. Ciencias Agrarias, Universidad Nacional. Docente-Investigador U.D.C.A, hasta Julio 2011; e-mail: mcabezasg@unal.edu.co
\end{abstract}

Rev. U.D.C.A Act. \& Div. Cient. 17(2): 381-390, Julio-Diciembre, 2014

RESUMEN

El trabajo se realizó en el municipio de Firavitoba - Boyacá, con el objeto de evaluar las respuestas de crecimiento y de desarrollo de Acca sellowiana en condiciones de riego y de sequía. Se utilizaron plantas cultivar Tibasosa, de tres años de edad, en buenas condiciones fitosanitarias y con similar tamaño. El riego, se aplicó por goteo, con goteros de $4 \mathrm{~L} \mathrm{~h}^{-1}$ y 2 goteros/planta. Se aplicó un diseño de bloques completos al azar, con tres bloques y 10 plantas por parcela. Dentro de cada bloque, se distribuyeron al azar cinco tratamientos correspondientes a diferentes niveles de riego: $100 \%$ (testigo), 75, 50, 25 y 0\%. Por cada planta, de manera directa, se contaron en cuatro ocasiones hasta el día 406, después de iniciar los tratamientos, el número de ramas, de hojas completamente formadas y de botones florales desarrollados. La altura, se midió desde el cuello de la raíz hasta la parte más alta de la planta; el área foliar $\left(\mathrm{cm}^{2}\right)$, se determinó con un medidor portátil de área foliar; el índice de área foliar (IAF), se calculó mediante la relación entre el área foliar y el área de suelo ocupado por la planta. Los datos obtenidos fueron sometidos a un análisis de varianza (ANAVA) y a la prueba de comparación de promedios de Tukey (5\%). El software utilizado fue SAS Ve. 9.2. Se determinó que el riego es fundamental para un buen desarrollo fisiológico de la feijoa, específicamente, para el desarrollo vegetativo de la planta, en especial, con factores de agotamiento entre el 50 y $75 \%$.

Palabras clave: Estrés hídrico, área foliar, crecimiento y desarrollo.

\section{SUMMARY}

The research was carried out at Firavitoba, Boyacá, to determine the growth responses and the plant development under irrigation and drought condition. Feijoa plants, three years old, in good phytosanitary conditions and with similar size were used. Irrigation was applied with drip emitters 4L and 2 h- 1 drippers / plant. A complete random block design was employed, with three blocks and 10 plants per plot. Within each block five treatments, corresponding to different irrigation levels $100 \%$ (control), $75 \%, 50 \%, 25 \%$ and $0 \%$ were randomly assigned. For each plant, four times, up to day 406 after treatment initiation, the number of new branches, of fully developed leaves and of floral buttons were counted directly. Height was measured from the root collar to the top of the plant; leaf area $\left(\mathrm{cm}^{2}\right)$ was measured with a portable leaf area meter; the leaf area index (LAI) was calculated by the ratio of leaf area and the soil area occupied by the plant. The data obtained were subjected to analysis of variance (ANOVA) and mean comparison test of Tukey (5\%). The software used was SAS Ve 9.2 (Cary, NC). It was determined that irrigation is essential for good physiological development of feijoa, specifically for the vegetative plant growth, specifically under $50-70 \%$ irrigation levels.

Key words: Hydric stress, leaf area, growth and development.

\section{INTRODUCCIÓN}

La feijoa (Acca sellowiana (O. Berg) Burret) (Myrtaceae) es originaria de Suramérica, de las zonas comprendidas entre 
el sur de Brasil, Uruguay, las partes altas del occidente de Paraguay y el nororiente de Argentina (Schumann \& Lüdders, 1992; CCI, 2008). El fruto ha tenido excelente aceptación en los mercados nacionales e internacionales, para consumo en fresco o para agroindustria, por lo cual, se ha convertido en necesidad para el agricultor contar con una producción constante, para lograr un abastecimiento al mercado (Combariza et al. 2007).

La planta, se caracteriza por su amplia capacidad de adaptación, encontrándose en Colombia cultivos comerciales en zonas con altitudes sobre 1.800 y 2.700 msnm, temperaturas entre $13^{\circ} \mathrm{C}$ y $21^{\circ} \mathrm{C}$ (promedio $16^{\circ} \mathrm{C}$ ), precipitación anual de 700 a $1.200 \mathrm{~mm}$, una exposición a la radiación de 1.500 horas brillo solar/año y humedad relativa promedio del $70 \%$ (Fischer, 2003; Quintero, 2003). En condiciones subtropicales y estacionales presenta una cosecha anual, mientras que en zonas tropicales puede producir, en forma continua, durante todo el año (Quintero, 2012).

La fisiología de los frutales perenes que se cultivan en el trópico tiene unas particularidades, derivadas de las respuestas de las especies al hidro-periodo, que hace referencia al efecto que tiene la humedad del suelo, producto del aporte de agua, sobre el desarrollo vegetal. Un estado poco favorable, como la sequía temporal, induce a la planta a diferenciar yemas florales, mientras que la humedad del suelo provoca posteriormente la emergencia y el crecimiento de flores y de frutos.

Tradicionalmente, los cultivos de feijoa se han establecido en zonas de ladera, entre los 1.200 y 2.400 msnm, con regímenes de lluvias monomodales, zona oriental de la cordillera oriental o bimodal, valles interandinos y zona central andina. Las dos zonas han hecho que las producciones de la fruta se aglomeren en dos épocas del año, junio y julio y noviembrediciembre, lo cual, provoca una alta estacionalidad de cosechas e inconvenientes en el mercadeo (Fischer, 2003).

La CCI (2008) ubica la feijoa "entre las 10 especies frutícolas agroindustriales promisorias en Colombia, pues se destaca por ser una fruta exótica, con gran potencial en los mercados extranjeros". Durante el 2010, se cosecharon en Colombia 147 ha de feijoa, con una producción total de 1.400t, siendo los principales departamentos productores Boyacá, Caldas, Cundinamarca, Santander y Norte de Santander (Agronet, 2012).

Debido a las expectativas comerciales del cultivo y a la importancia nutricional y medicinal de este fruto es importante optimizar el manejo agronómico del mismo (Rodríguez et al. 2010); actualmente, se siembra teniendo en cuenta las investigaciones realizadas en otros países.
Un déficit hídrico afecta negativamente las funciones fisiológicas, como fotosíntesis, respiración, reacciones metabólicas y anatómicas, crecimiento, reproducción, desarrollo de semillas, absorción de nutrientes minerales, transporte de asimilados y producción (Hsiao, 1973; Quiñones et al. 2007; Casierra \& Rodríguez, 2006; García-Sánchez et al. 2003; Ortuño et al. 2004). El efecto dependerá de la intensidad de la sequía, de la duración de la misma y de la época en que ocurre dentro del ciclo del cultivo; esto ocurre tanto en cítricos como en otros frutales (Ballester et al. 2011).

A pesar que la feijoa es considerada una fruta de importancia en los mercados nacional e internacional, debido a sus cualidades nutritivas, en Colombia, no se han desarrollado trabajos de investigación tendientes a obtener información acerca de los requerimientos y las exigencias hídricas del cultivo, así como determinar los efectos del déficit hídrico sobre el crecimiento, desarrollo y rendimiento, objetivo de este estudio.

\section{MATERIALES Y MÉTODOS}

El trabajo experimental, se realizó en el municipio de Firavitoba (Boyacá), a final del segundo semestre de 2011 y el primer y segundo semestre de 2012 , a $5^{\circ} 39^{\prime} 50^{\prime \prime}$ de latitud Norte, $74{ }^{\circ} 5^{\prime} 10$ ” longitud Occidental, a 2450 msnm de altura, humedad relativa de $70 \%$, temperatura promedio anual de $15^{\circ} \mathrm{C}$, precipitación de $850 \mathrm{~mm}$, distribuidos en régimen bimodal de lluvias. Allí, se presentan dos periodos secos bien marcados: el primero, entre diciembre y marzo y, el segundo, entre julio y agosto.

Se utilizaron plantas de feijoa cultivar Tibasosa, de tres años de edad, sembradas a distancias de 3 x $3 \mathrm{~m}$. Las plantas seleccionadas estaban en buenas condiciones fitosanitarias, con similar tamaño. El riego, se aplicó mediante sistema de riego por goteo, con goteros de $4 \mathrm{~L} \mathrm{~h}^{-1}$ y 2 goteros/planta.

Se empleó un diseño de bloques completos al azar, con tres bloques; el criterio de bloque correspondió al relieve y dentro de cada bloque, se distribuyeron al azar cinco tratamientos, correspondientes a diferentes niveles de riego; estos fueron 100, 75, 5025 y 0\% (testigo) del valor, obtenido mediante la fórmula 1. Las unidades experimentales fueron parcelas de 10 plantas.

Después de seleccionar las plantas, se les realizó una poda de homogenización e, inmediatamente, se inició la aplicación de los tratamientos de riego. La lámina de agua a aplicar, se determinó por medio de la siguiente fórmula:

$$
\text { Lámina de agua }=\frac{\mathrm{CC}-\mathrm{PMP}}{100} * \mathrm{Gs} * \mathrm{Pr} * 1
$$

Donde:

CC $=$ Capacidad de Campo 
PMP = Punto de Marchitez Permanente

Gs $=$ Gravedad específica

$\operatorname{Pr}=$ Profundidad de raíces

$1=$ Corresponde al coeficiente de agotamiento

Se determinaron, a nivel de laboratorio, algunas características hidro-físicas del suelo, principalmente, las tensiones y la densidad aparente, que permitiera determinar la lámina de agua disponible, con el fin de realizar aplicaciones de riego, modificando los factores de agotamiento, es decir, los tratamientos.

Para evitar que el agua de escorrentía afecte las parcelas, se hicieron canales de $50 \mathrm{~cm}$ de profundidad, para separar las unidades experimentales. Las diferentes labores culturales y manejo del cultivo, se hicieron de acuerdo al manejo tradicional de los agricultores de la zona.

Los tratamientos, se aplicaron en alrededor de 406 días. Las mediciones de las principales variables ecofisiológicas (longitud de la planta, número ramas, hojas y botones florales), se realizaron a los 45, 263, 320 y 406 días, después del inicio de los tratamientos.
Las variables correspondieron al número de ramas, de hojas y de botones florales, que se tomaron mediante conteo directo sobre cada una de las plantas de cada tratamiento por parcela. Además, se midió la altura desde el cuello de la raíz hasta la parte más alta de la planta, mediante un flexómetro. El área foliar $\left(\mathrm{cm}^{2}\right)$, se determinó con un medidor portátil de área foliar y el índice de área foliar (IAF), se calculó mediante la relación entre el área foliar y el área de suelo ocupado por la planta.

Los datos obtenidos fueron sometidos a un análisis de varianza (ANAVA) y a la prueba de comparación de promedios de Tukey (5\%). El software utilizado fue SAS Ve. 9.2 (Cary, N.C).

\section{RESULTADOS Y DISCUSIÓN}

Número de ramas: Aumentó en función del tiempo en todos los tratamientos, excepto en las plantas de $75 \%$ del riego, que presentaron una leve disminución; sin embargo, no se manifestaron diferencias estadísticas en ningún momento de muestreo (Figura 1 A).

A.

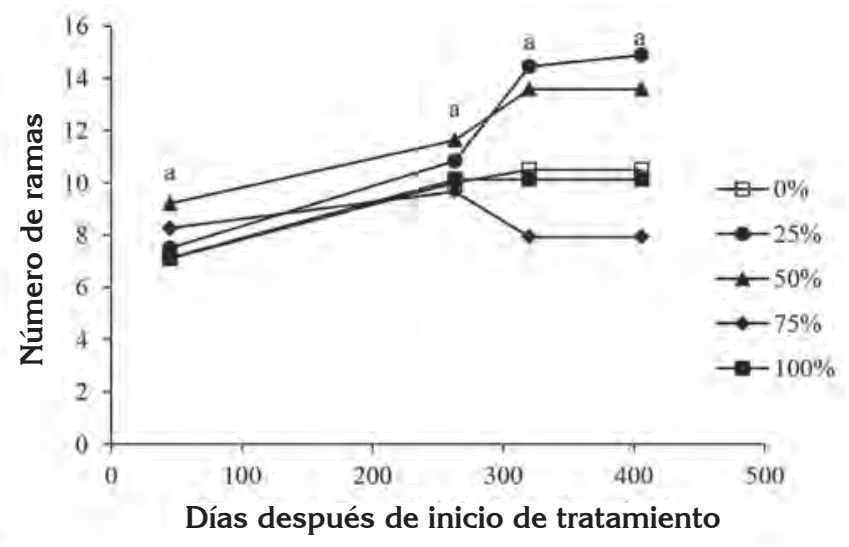

:

B.

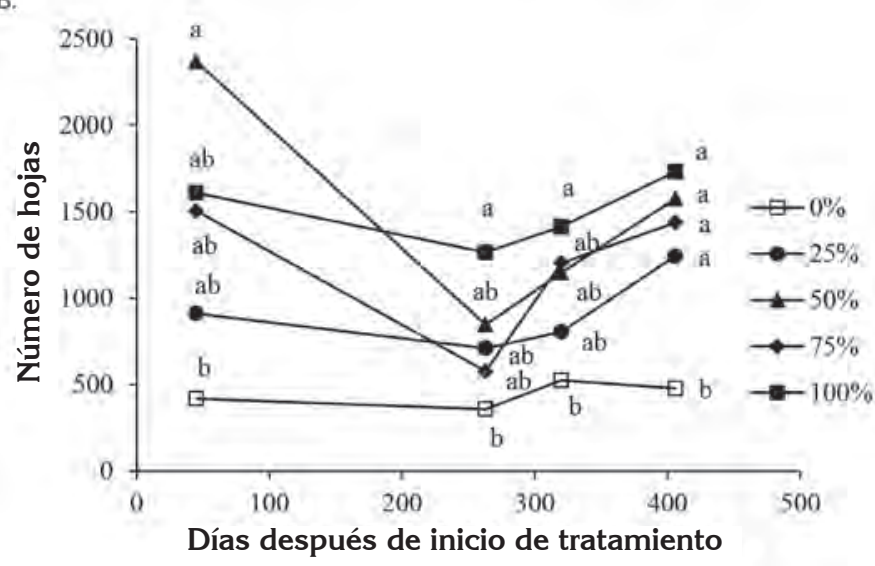

Figura 1: Efecto del riego y del déficit hídrico sobre: A) Número de ramas; B) Número de hojas de plantas de feijoa. Promedios seguidos de diferentes letras en el mismo punto de muestreo presentan diferencias estadísticas, según la prueba de Tukey (5\%). 
Se esperaba que el mayor número de ramas se diera en las plantas con mayor altura, por poseer más brotes vegetativos, que se convierten en ramas, situación similar a la encontrada en plantas jóvenes de guayaba, por Cañizares et al. (2003); sin embargo, esta situación no se observó en este experimento, posiblemente, porque se requiere más tiempo de exposición a los tratamientos, para encontrar una respuesta diferencial.
De otra parte, también se evidencia que, a pesar de que existen diferencias numéricas representativas, no se observan disimilitudes estadísticas en el número de ramas. Esta situación pone de manifiesto que, al parecer, las plantas de feijoa responden de manera diferente a la misma condición de riego, tal vez por variabilidad genética (Tabla 1).

Tabla 1. Coeficiente de variación de variables ecofisiológicas para los diferentes tratamientos.

\begin{tabular}{|c|c|c|c|c|}
\hline & \multicolumn{3}{|c|}{ CV (\%) } \\
\hline TRATAMIENTO & RAMAS & ALTURA & HOJAS & BOTON FLORAL \\
\hline 0 & 39,43 & 8,62 & 18,84 & 1644,13 \\
\hline 25 & 10,51 & 5,77 & 18,55 & 37,08 \\
\hline 50 & 10,51 & 5,08 & 11,56 & 41,25 \\
\hline 75 & 16,55 & 6,06 & 14,95 & 72,64 \\
\hline 100 & 23,67 & 9,21 & 32,32 & 316,15 \\
\hline
\end{tabular}

Siendo así, Barlow et al. (1980) mencionan que la iniciación, así como la diferenciación de las estructuras vegetativas y reproductivas y el alargamiento celular, una vez diferenciadas las células, es muy sensible al estrés hídrico y, al igual que los procesos fisiológicos, no es afectada necesariamente con la misma intensidad. Lo anterior, se puede deber a que en condiciones de alta concentración de solutos, las células ejercen presión de turgencia contra las paredes celulares, lo que soporta el crecimiento. Cuando las células pierden turgencia, no llevan a cabo el alargamiento y expansión y, si esto ocurre por periodos largos de tiempo, la planta se deshidrata y muere (Zyalalov, 2004).

Bajo las condiciones del estudio, el número de ramas en las plantas de feijoa no se afectó significativamente, por los diferentes niveles de riego.

Número de hojas: Se presentaron diferencias significativas en todos los puntos de muestreo. A los 45 días después de tratamiento (DDT), el mayor número de hojas (2.369), se obtuvo con $50 \%$ de riego; de ahí en adelante, el mayor número de hojas, se generó con la aplicación del 100\% de riego. Las plantas del testigo, como era de esperarse, presentaron el menor número de hojas, que en promedio estuvieron por debajo de 550 (Figura 1B).

El menor número de hojas encontrado en las plantas de feijoa, con $0 \%$ de riego, puede tener varias explicaciones, siendo dos las principales. La primera tiene relación con la abscisión de hojas y, la segunda, se explica por menor emisión de nuevas hojas. La ausencia de riego ocasiona desbalance hormonal en muchas especies vegetales, dando lugar a alteraciones significativas en los procesos fisiológicos del crecimiento y del desarrollo. Adicionalmente, la ausencia de riego reduce el contenido de ácido indolacético (Darbyshire, 1971) y se presentan evidencias claras de inhibición del transporte de auxinas por el déficit hídrico, lo que puede provocar la abscisión de determinados órganos de la planta (Davenport et al. 1977). Desde el punto de vista de producción, los mayores efectos del estrés hídrico en la síntesis del etileno, se manifiestan en la senescencia foliar y la abscisión de hojas.

La senescencia, la abscisión y la caída de las hojas son medios drásticos para limitar la transpiración, en caso de sequía severa; sin embargo, esta adaptación conlleva un retraso importante en el crecimiento y en el desarrollo de la planta, como lo comprobaron Gómez-Cadenas et al. (1996), en mandarina "Clementina de Nules". Las hojas jóvenes tienen potenciales osmóticos mayores que las hojas viejas y, consecuentemente, pierden la turgencia antes que las hojas maduras. Así pues, el crecimiento de hojas nuevas es muy sensible al déficit hídrico (Syvertsen, 1985), que se ajusta a las condiciones locales en el periodo de estudio, básicamente, con disminuciones en volúmenes de precipitación respecto a la normal, de alrededor del $22 \%$, incluso, en el departamento de Boyacá, que alcanzó diferencias del $87 \%$.

La emisión de nuevas hojas desaparece por completo durante periodos prolongados sin riego, pero al reanudarlo, rápidamente se inicia una intensa brotación y el crecimiento vegetativo se recupera (González-Altozano \& Castel, 2003). 
Altura: Aumentó durante el tiempo del experimento. Hubo diferencias estadísticas significativas en todos los puntos de muestreo, donde las plantas con $0 \%$ riego presentaron la menor altura, pasando de 0,76 a 0,95m, mientras que las plantas que fueron tratadas con el $50 \%$ del riego, tuvieron la mayor altura y al final del experimento alcanzaron los 1,26 m (Figura 2A).
Un buen contenido de agua genera células más alargadas, debido a la presión de turgencia (Salisbury \& Ross, 2000; Marschner, 2002). Montenegro \& Malagón (1990) afirman que cuando las plantas disponen de un buen contenido de agua disminuyen el gasto energético que emplean para su crecimiento, razón por la cual, las plantas de feijoa que presentaron mayor altura fueron las de un suministro hídrico adecuado, es decir los factores de agotamiento entre 50 a $75 \%$.

A.

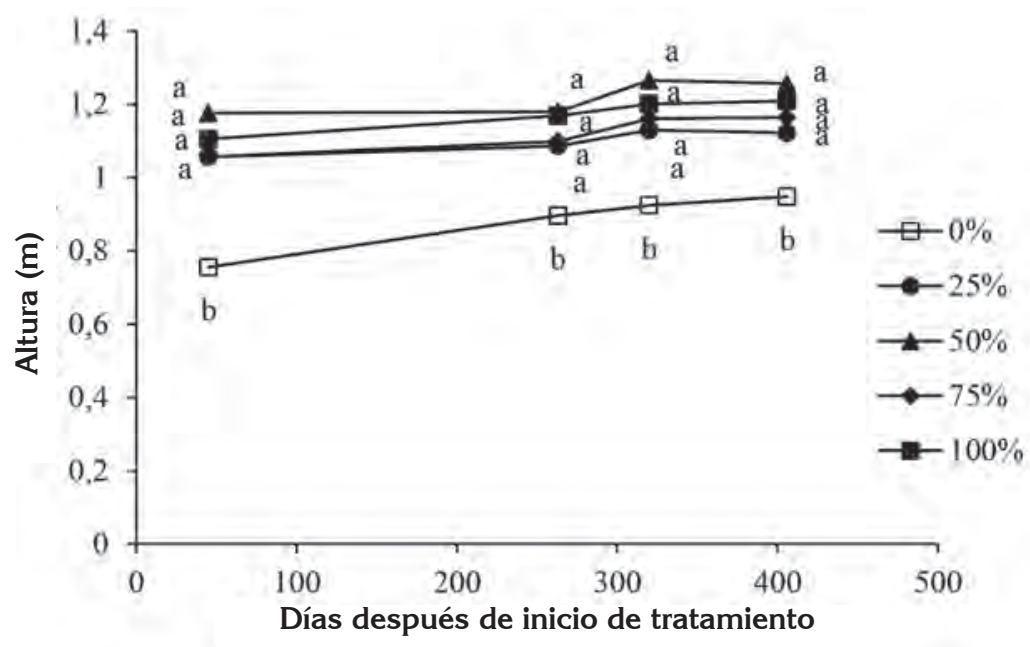

B.

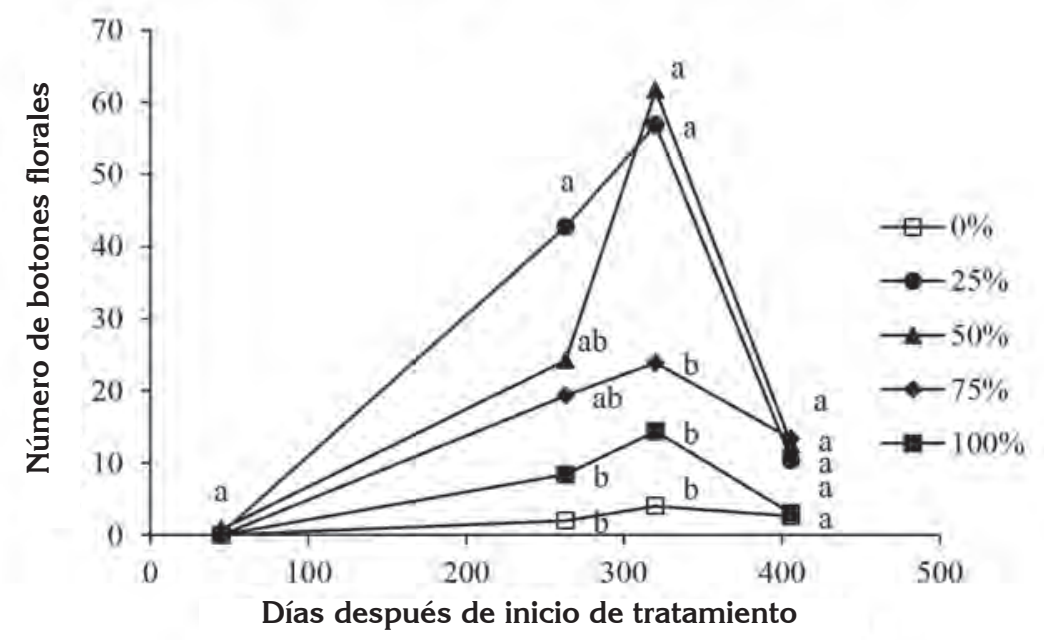

Figura 2: Efecto del riego y del déficit hídrico sobre: A) Altura de plantas de feijoa; B) Número de botones florales de plantas de feijoa. Promedios seguidos de diferentes letras en el mismo punto de muestreo presentan diferencias estadísticas, según la prueba de Tukey (5\%).

Además, Sánchez-Blanco et al. (2004) mencionan que tratamientos con déficit hídrico en romero mostraron una disminución en la altura, debido a que bajos niveles de agua disminuyen la presión de turgor de la célula y la pared celular no se expande, lo que genera un menor crecimiento celular (Marschner, 2002) y, a su vez, una altura reducida, lo cual concuerda con lo encontrado en este estudio. 
La expansión celular, se debe al potencial de turgencia, suministro de sales minerales, agua y materia elaborada (Taiz \& Zeiger, 2006); debido a esto, el crecimiento es uno de los procesos más afectados por el déficit hídrico. Cítricos regados por debajo de sus requerimientos no alcanzan el tamaño de las plantas que sí reciben un suministro adecuado (García-Orellana et al. 2007), situación similar a lo encontrado en este estudio.

Número de botones florales: Se observó un aumento de los botones florales entre los 45 días después del inicio del tratamiento, hasta los 320 después; luego, hubo una disminución representativa hasta los 406 días. Con diferencias estadísticas, las plantas con 25 y $50 \%$ de riego presentaron el mayor número de botones florales, hasta los 320 DDT; en el último muestreo (406 DDT), no indicó diferencias significativas. Durante todo el experimento, las plantas con $0 \%$ de riego tuvieron la menor cantidad de botones florales (Figura 2B); lo anterior, se debe a que el déficit hídrico inhibe la fotosíntesis y disminuye el flujo de fotosintatos a los órganos en desarrollo (Boyer \& Westgate, 2004). Se afectan las flores y la fecundación produciendo un aborto floral y abscisión del fruto (González-Altozano \& Castel, 2003). Cuando el estrés ocurre durante el desarrollo del cigoto, se provoca absorción o bien, el saco embrionario puede ser afectado en su desarrollo, entre un 15 y $43 \%$, induciendo una asincronía entre la antesis y la floración femenina, en plantas de feijoa.

En cítricos, se ha encontrado que un déficit hídrico de alrededor de un $40 \%$ de las necesidades de agua, produce una disminución del crecimiento de brotes y del tamaño de los frutos, pero se observa un aumento en el número de flores por árbol. Esto puede explicar el alto número de botones florales registrados en las plantas de feijoa con riego del 25, 50 y $75 \%$. En el trópico, el principal factor inductor de la floración es el estrés hídrico; éste regula la época, la intensidad, la duración y la distribución de la floración (Davenport, 1990; Casierra \& Rodríguez, 2006). Con el déficit hídrico cesa el crecimiento de los tallos y del sistema radical; además, dependiendo de la intensidad del estrés, se puede presentar marchitez de la hoja, disminución de la conductancia estomática, de la asimilación neta de $\mathrm{CO}_{2}$ y de la conductividad radical (Davies \& Albrigo, 1994). En este periodo, las yemas vegetativas desarrollan la capacidad para florecer. En el proceso de inducción floral, se incluyen los eventos que suceden en la transición del crecimiento vegetativo a la producción de inflorescencias (Davenport, 1990).

En cuanto a la exigencia hídrica de las plantas de feijoa, la distribución de lluvias durante la estación de crecimiento de las plantas influye en los estadios de floración y de cuajado de los frutos (Seymor et al. 1993; Pachón \& Quintero, 1993). Schuman \& Ludders (1992) mencionan que los suministros adecuados de agua influyen de manera directa sobre el cre- cimiento, el desarrollo y la productividad del cultivo de feijoa. La respuesta de los frutales a estrés hídrico es la reducción en el crecimiento y ocurrencia precoz de la senescencia del cultivo. El tamaño y la calidad del fruto dependen de los suministros adecuados y regulares de agua. En períodos secos prolongados, la planta reacciona con caída de hojas, de flores y de frutos. En feijoa, los mejores resultados en floración, en polinización y en llenado del fruto, se presentan con libre exposición a la radiación solar, siempre y cuando no existan condiciones secas y temperaturas altas (Fischer, 2003).

Área foliar: Se observó una disminución del área foliar hasta los 263 DDT, después hubo un aumento hasta el final del experimento, principalmente, en aquellos tratamientos con riego. Se evidenciaron diferencias significativas en todas las épocas de muestreo, donde las plantas, con $0 \%$ de riego, mostraron la menor área foliar, mientras que las plantas con los demás tratamientos fueron estadísticamente superiores, pero no presentaron diferencias entre ellas (Figura 3A).

Posiblemente, las plantas de feijoa sometidas a estrés hídrico ( $0 \%$ de riego), cierran estomas para disminuir la pérdida de agua, pero esta situación genera menor entrada de $\mathrm{CO}_{2}$ para la fotosíntesis, por lo cual, el crecimiento de la planta, incluyendo la expansión del área foliar, se pueden ver reducidas; no obstante, en las plantas de feijoa con suministros moderados de agua, es decir, con 25,50 y $75 \%$, los resultados son bastante favorables, porque el área foliar es similar al obtenido con el $100 \%$ de riego; esto pone en evidencia que las plantas de feijoa presentan ciertos mecanismos de tolerancia al déficit hídrico. Al respecto, cuando el déficit hídrico se desarrolla lentamente, se dan cambios en procesos de desarrollo que tienen varios efectos sobre el crecimiento. Uno, de principal importancia, es la limitación específica de la expansión foliar; aunque el área foliar es importante, pues de ella depende la fotosíntesis, una rápida expansión foliar puede afectar negativamente la adaptación a la poca disponibilidad de agua (Moreno, 2009). Otro mecanismo de resistencia a nivel fisiológico es el cierre de estomas, ya que son los responsables de la mayor proporción de pérdida de agua en las plantas; esta respuesta está mediada por la hormona ácido abscísico (Taiz \& Zeiger, 2006)

Diferentes autores coinciden en que la sequía afecta, en general, el crecimiento y el desarrollo de las plantas (Outtar et al. 1987; NeSmith \& Ritchie (1992) y ha relacionado estrechamente la reducción del área foliar con reducción del rendimiento. Un riego deficitario puede generar una reducción del vigor vegetativo de los árboles frutales; se disminuye el área foliar, pero se aumenta la entrada de luz al interior del árbol, de forma que los rendimientos de fruta se mantienen o, incluso, aumentan (Romero et al. 2004; Fereres \& Soriano, 2007). 
A.

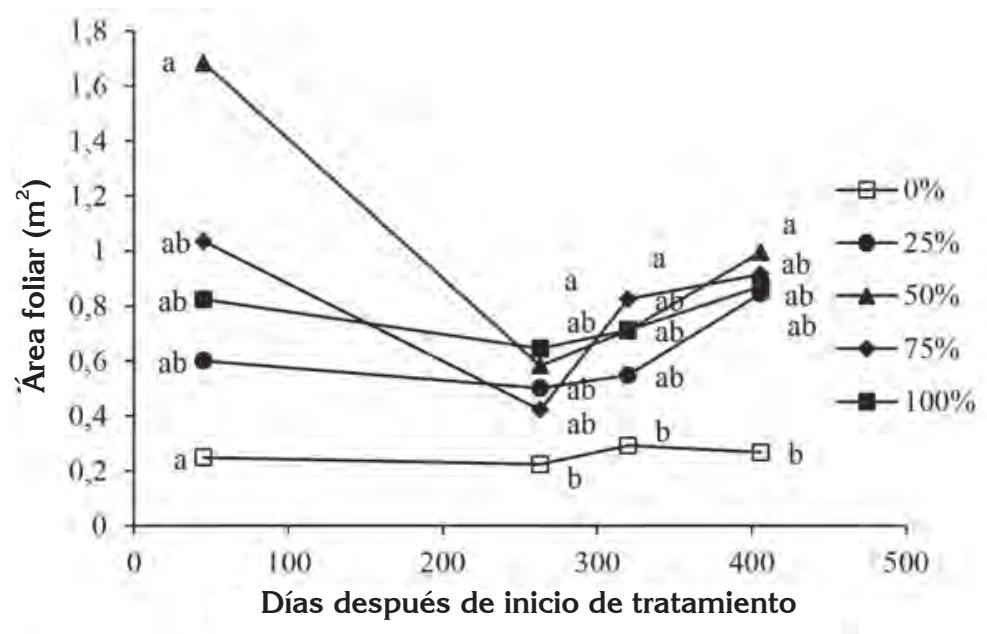

B.

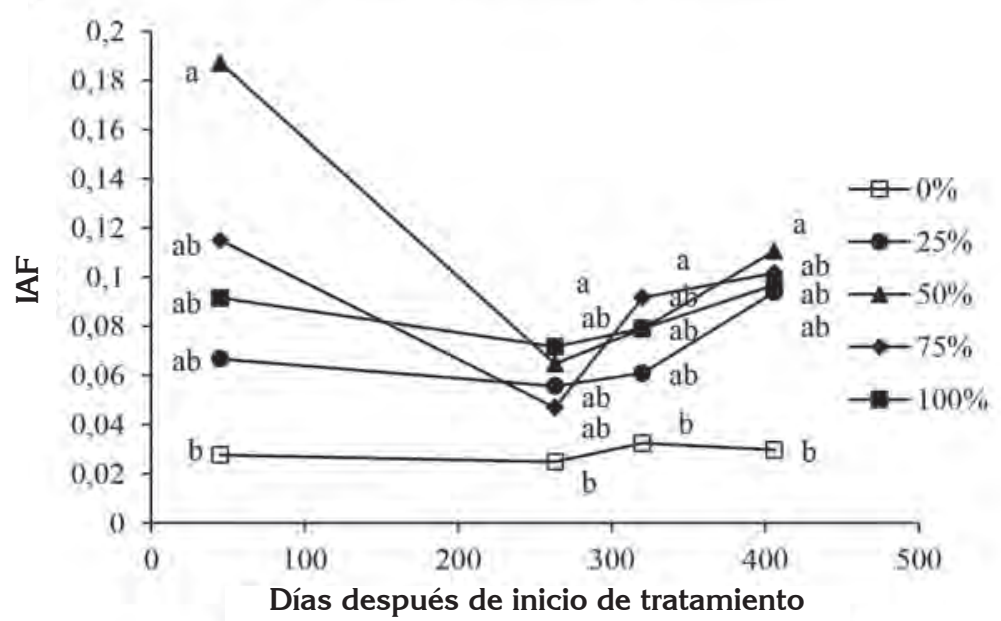

Figura 3: Efecto del riego y del déficit hídrico sobre: A) Área foliar; B) Índice de Área Foliar de plantas de feijoa. Promedios seguidos de diferentes letras en el mismo punto de muestreo presentan diferencias estadísticas, según la prueba de Tukey (5\%).

La adaptación al estrés hídrico puede ser de varios tipos, dependiendo de los niveles de dicho estrés. Estas adaptaciones combinan factores anatómicos y fisiológicos, que limitan el almacenamiento y el transporte del agua por la planta. Como consecuencia del estrés hídrico, se producen una serie de respuestas, como: disminución en la conductancia de los estomas, con lo cual, se evita la pérdida de agua por transpiración y abscisión foliar (Pérez-Pérez et al. 2008), disminuyendo el área foliar y, por tanto, la superficie de transpiración y de adaptaciones, a nivel metabólico y osmoregulación; en las células acumulan una serie de solutos compatibles con el funcionamiento normal del mecanismo celular (Andreev, 2001; Barry et al. 2004; Navarro et al. 2010). Cuando el déficit hídrico se presenta lentamente, las plantas pueden exhibir respuestas de aclimatación, que tienen efectos sobre el crecimiento, como la disminución de la expansión foliar y el aumento del crecimiento radicular (Shao et al. 2008).

Índice de área foliar: Los valores obtenidos estuvieron entre 0,02 y 0,19 . Se observaron diferencias estadísticas en todos los muestreos. El menor valor de IAF, en todo el estudio, se obtuvo en las plantas con $0 \%$ de riego; por su parte, las plantas con el $50 \%$ de riego fueron las que presentaron el mayor IAF durante la mayor parte del tiempo, en que se realizó el experimento (Figura 3B).

El índice de área foliar es el área proyectada de las superficies de las hojas por unidad de área de suelo (Marshall \& Waring, 1986; De la Casa et al. 2007). Aunque esta medida se vincula con el crecimiento y el desarrollo, también es una 
medida ecofisiológica, ya que se relaciona con el área de la superficie fotosintética y de la transpiración dentro del dosel (Chen et al. 1997) y está fuertemente conectada con la productividad de cultivos aprovechables, debido a que determina la habilidad para capturar la energía lumínica (Wilhem et al. 2000). En este sentido, las plantas con $0 \%$ de riego tienen menor capacidad para captar luz y, por ende, sus tasas de fotosíntesis son más bajas, mientras que las plantas de feijoa con suministro hídrico tienen mayor área foliar por unidad de suelo, que les permite mayor captación de energía lumínica, mayor capacidad de producción de fotoasimilados, que conlleva a generar plantas con mayor crecimiento, tal como se evidencia en las demás variables, medidas en esta investigación.

El número de hojas en plantas de feijoa es un aspecto ecofisiológico que responde diferencialmente a los diferentes niveles de riego; de manera general, a medida que se disminuyó el riego también lo hizo el número de hojas, así que con $100 \%$ de riego, el número de hojas fue el mayor, mientras que con $0 \%$, la cantidad de hojas fue el más bajo, presentando coherencia con los resultados de IAF.

La altura de la planta de feijoa no se vio afectada significativamente por niveles moderados y suficientes de riego (25, 50,75 y $100 \%$ de riego), pero fue mucho menor cuando no se le aplicó riego.

El número de botones florales fue mayor con 25 y $50 \%$ de riego, mientras que con niveles más altos o más bajos de suministro hídrico, los botones florales disminuyeron, siendo mayor esta disminución con $0 \%$ de riego, debido a que el déficit hídrico afecta directamente el desarrollo de órganos reproductivos.

El área foliar y el índice de área foliar son parámetros sensibles a la ausencia de riego, pero con niveles moderados de riego es suficiente para tener valores estadísticamente iguales a los del nivel de $100 \%$ de riego.

Se recomienda aplicar $100 \%$ de riego a las plantas de feijoa para que presenten un mejor comportamiento ecofisiológico, en el municipio de Firavitoba (Boyacá), aunque por costos de riego, se podrían usar láminas del 50 y del $75 \%$, ya que son factores de agotamiento, que permiten que la planta tenga un adecuado crecimiento y desarrollo.

Conflicto de intereses: El manuscrito fue preparado y revisado por el autor, quien declara que no existe ningún conflicto de intereses, que ponga en riesgo la validez de los resultados presentados.

\section{BIBLIOGRAFÍA}

1. AGRONET. 2012. Reportes Estadísticos. Disponible desde Internet en: http://www.agronet.gov.co (con acceso 15/08/2012).

2. ANDREEV, I. 2001. Functions of the vacuole in higher plants cells. Russian J. Plant Physiol. 48(5):777-787.

3. BALLESTER, C.; CASTEL, J.; INTRIGLIOLO, D.S.; CASTEL, J.R. 2011. Response of Clementina de Nules citrus trees to summer deficit irrigation. Yield components and fruit composition. Agr. Water Managem. 98:1027-1032.

4. BARLOW, E.W.R.; MUNNS, R.E.; BRADY, J.C. 1980. Drought responses of apical meristems. En: Turner, N.C.; Kramer, P.J. (eds). Adaptation of plants to water and high temperature stress. Nueva York: Wiley Interscience. p.191-205.

5. BARRY, G.H.; CASTLE, W.S.; DAVIES, F.S. 2004. Rootstocks and plant water relations affect sugar accumulation of citrus fruit via osmotic adjustment. J. Am. Soc. Hort. Sci. 129:881-889.

6. BOYER, J.S.; WESTGATE, M.E. 2004. Grain yields with limited water. J. Exp. Bot. 55(407):2385-2394.

7. CAÑIZARES, A.; LAVERDE, D.; PUESME, R. 2003. Crecimiento y desarrollo del fruto de guayaba (Psidium guajava L.), en Santa Bárbara, Estado de Monagas Venezuela. Rev. UDO Agrícola. 3(1):34-38.

8. CASIERRA, F.; RODRÍGUEZ, S. 2006. Tolerancia de plantas de feijoa (Acca sellowiana Berg Burret) a la salinidad por $\mathrm{NaCl}$, en Colombia, Agr. Col. 24(2):258265.

9. CORPORACIÓN COLOMBIA INTERNACIONAL -CCI-. 2008. Producción y comercialización de la feijoa en Colombia. Disponible desde internet en: http://www. cci.org.co/publicaciones (con acceso 09/11/2010).

10. CHEN, J.M.; RICH, P.M.; GOWER, S.T.; NORMAN, J.M; PLUMMER, S. 1997. Leaf area index of boreal forests: theory, techniques and measurements. J. Geophys. Res. 102:429-443.

11. COMBARIZA L., C.; NEIRA, G.; FISCHER, G.; CORREDOR, Y.; QUINTERO, C. 2007. Crecimiento, producción y calidad de fruta en feijoa [Acca sellowiana (O.Berg) Burret] en respuesta al nitrato de potasio, 
fosfato de potasio y ethephon. Rev. Col. Cienc. Hort. 1(2):170-181.

12. DARBYSHIRE, B. 1971. Changes in indolacetic acid oxidase activity associated with plant water potential. Physiol. Plant. 25(1):80-84.

13. DAVENPORT, T.L.; MORGAN, P.W.; JORDAN, W.R. 1977. Auxin transport as related to leaf abscission during water stress in cotton. Plant Physiol. 59:554-557.

14. DAVENPORT, T.L. 1990. Citrus flowering. Hort. Rev. 12:349-408.

15. DAVIES, F.S.; ALBRIGO, L.G. 1994. Citrus. CAB International, Wallingford, U.K. 254p.

16. DE LA CASA, A.; OVANDO, G.; BRESSANINI, L.; RODRÍGUEZ, A.; MARTÍNEZ, J. 2007. Uso del índice de área foliar y del porcentaje de cobertura del suelo para estimar la radiación interceptada en papa, en Argentina. Agr. Técn. (Chile) 67(1):78-85.

17. FERERES, E.; SORIANO, M.A. 2007. Deficit irrigation for reducing agricultural water use. J. Exp. Bot. 58(2):147-159.

18. FISCHER, G. 2003. Ecofisiología, crecimiento y desarrollo de la feijoa. En: Fischer, G.; Miranda, D.; Cayón, G.; Mazorra, M. (eds). Cultivo, poscosecha y exportación de la feijoa (Acca sellowiana Berg). Produmedios, Bogotá. p.9-26.

19. GARCÍA-ORELLANA, Y.; RUIZ-SÁNCHEZ, M.C.; ALARCÓN, J.J.; CONEJERO, W.; ORTUÑO, M.F.; NICOLÁS, E.; TORRECILLAS, A. 2007. Preliminary assessment of the feasibility of using maximum daily trunk shrinkage for irrigation scheduling in lemon trees. Agr. Water Managem. 89:167-171.

20. GARCÍA-SÁNCHEZ, F.; CARVAJAL, M.; PORRAS, I.; BOTÍA, P.; MARTÍNEZ, V. 2003. Effects of salinity and rate of irrigation on yield, fruit quality and mineral composition of 'Fino 49' lemon. Eur. J. Agron. 19:427-437.

21. GÓMEZ-CADENAS, A.; TADEO, F.R.; TALON, M.; PRIMO-MILLO, E. 1996. Leaf abscission induced by ethylene in water stressed intact seedling of Cleopatra mandarin requires previous abscisic acid accumulation in roots. Plant Physiol. 112:401-408.

22. GONZÁLEZ-ALTOZANO, P.; CASTEL, J.R. 2003. Riego deficitario controlado en Clementina de Nules. II
Efectos sobre el crecimiento vegetativo. Spanish J. Agr. Res. 1(2):93-101.

23. HSIAO, T.C. 1973. Plant responses to water stress. Ann. Rev. Plant Physiol. 24:519-570.

24. MARSCHNER, H. 2002. Mineral nutrition of higher plants. $2^{a}$ ed., London., Academic Press. 889p.

25. MARSHALL, J.D.; WARING, R.H. 1986. Comparison of methods of estimating leaf area index in old-growth douglar fir. Ecol. 67:975-979.

26. MONTENEGRO, G.; MALAGON, D. 1990. Propiedades físicas de los suelos. Bogotá. IGAC. 813p.

27. MORENO, L. 2009. Respuesta de las plantas al estrés por déficit hídrico. Una revisión. Agr. Col. 27(2):179191.

28. NAVARRO, J.M.; PÉREZ-PÉREZ, J.G.; ROMERO, P.; BOTÍA, P. 2010. Analysis of the changes in quality in mandarin fruit, produced by deficit irrigation treatments. Food Chem. 119:1591-159.

29. NESMITH, D.S.; RITCHIE, J.T. 1992. Maize (Zea mays L.) response to a severe soil water-deficit during grain-filling. Field Crops Res. 29:23-35.

30. ORTUÑO, M.F.; ALARCÓN, J.J.; NICOLÁS, E.; TORRECILLA, A. 2004. Interpreting trunk diameter changes in young lemon trees under deficit irrigation. Plant Sci. 167:275-280.

31. OUTTAR, S.R.; JONES, J.; CROOKSTON, R.K.; KAJEIOU, M. 1987. Effect of drought on water relations of developing maize kernels. Crop Sci. 27:730-735.

32. PACHÓN, G.; QUINTERO, O. 1993. La feijoa (Feijoa sellowiana Berg) fruta promisoria para Colombia. Acta Hort. 310:239-248.

33. PÉREZ-PÉREZ, J.G.; ROMERO, P.; NAVARRO, J.M.; BOTÍA, P. 2008. Response of sweet orange cv 'Lane late' to deficit irrigation in two rootstocks. I: Water relations, leaf gas exchange and vegetative growth. Irrigation Sci. 26(5):415-425.

34. QUINTERO, O. 2003. Selección de cultivares, manejo del cultivo y regulación de cosecha de feijoa. En: Fischer, G., Miranda, D.; Cayón, G.; Mazorra, M. (eds). Cultivo, poscosecha y exportación de la feijoa (Acca sellowiana Berg). Produmedios, Bogotá. p.49-71. 
35. QUINTERO, O.C. 2012. Feijoa (Acca sellowiana Berg). En: Fischer, G. (ed.). Manual para el cultivo de frutales en el trópico. Produmedios, Bogotá. p.443-473.

36. QUIÑONES, A.; MARTÍNEZ-ALCÁNTARA, B.; LEGAZ, F. 2007. Influence of irrigation system and fertilization management on seasonal distribution of $N$ in the soil profile and on N-uptake by citrus trees. Agr.: Ecosyst. Environm. 122:399-409.

37. RODRÍGUEZ, M.; ARJONA, H.; FISCHER, G.; CAMPOS, H.; CHAPARRO, M. 2010. Aspectos anatómicos del desarrollo del fruto de feijoa [Acca sellowiana (O.Berg) Burret]. Rev. Fac. Nal. Agr. Medellín. 63(1):5267-5273.

38. ROMERO, P.; BOTIA, P.; GARCIA F. 2004. Effects of regulated deficit irrigation under subsurface drip irrigation conditions on vegetative development and yield of mature almond trees. Plant Soil. 260:169-181.

39. SALISBURY, F.; ROSS, C. 2000. Fisiología de las Plantas. Vol 3. PARANINFO. 988p.

40. SÁNCHEZ-BLANCO, M.J.; FERNÁNDEZ, T.; NAVARRO, A.; BAÑON, S.; ALARCÓN, J. 2004. Effects of irrigation and air humidity preconditioning on water relations, growth and survival of Rosmarinus officinalis plants during and after transplanting. J. Plant Physiol. 161(10):1133-1142.
41. SCHUMANN, M.; LÜDDERS, P. 1992. Cultivation of feijoa: possibilities and limits in Colombia. Erwerbsobstbau. 34(4):110-112.

42. SEYMOR, G.B.; TUCKER, J.E.; TUCKER, G.A. 1993. Biochemistry of fruit ripening. Chapman \& Hall, Londres. p.173-174.

43. SHAO, H.B.; CHU, L.Y.; JALEEL, C.A.; ZHAO, C.X. 2008. Water-deficit stress-induced anatomical changes in higher plants. C.R. Biol. 331:215-225.

44. SYVERTSEN, J.P. 1985. Integration of water stress in fruit trees. Hort. Science. 20 (6):1039-1043.

45. TAIZ, L.; ZEIGER, E. 2006. Plant Physiology. 4th ed. Sinauer Associates, Sunderland, MA. 764p.

46. WILHEM, W.W.; RUWE, K.; SCHLEMMER, M. 2000. Comparison of three leaf area index meters in a crop canopy. Crop Sci. 40:1179-1183.

47. ZYALALOV, A. 2004. Water flows in higher plants: physiology, evolution, and system analysis. Russian J. Plant Physiol. 51(4):547-555.

Recibido: Junio 12 de 2014

Aceptado: Septiembre 23 de 2014

Como citar:

Peña Baracaldo, F.J.; Cabezas Gutiérrez, M. 2014. Aspectos ecofisiológicos de la feijoa (Acca sellowiana Berg) bajo condiciones de riego y déficit hídrico. Rev. U.D.C.A Act. \& Div. Cient. 17(2): 381-390. 